\title{
Secreted Phosphoprotein 1 (SPP1) Promotes Angiogenesis of Glioblastoma Through Up- regulating PSMA Expression Via Transcription Factor HIF-1a
}

\section{Yang Gao ( $\sim$ baiyizhanshi@126.com )}

Fudan University Shanghai Cancer Center

Wenjing Tu

Fudan University Shanghai Cancer Center

Hui Zheng

Tongji University

Liangdong Li

Fudan University Shanghai Cancer Center

Changshuai Zhou

Fudan University Shanghai Cancer Center

Mingtao Feng

Fudan University Shanghai Cancer Center

Lei Chen

Fudan University Shanghai Cancer Center

Deheng Li

Fudan University Shanghai Cancer Center

Xin Chen

Fudan University Shanghai Cancer Center

\section{Bin Hao}

Fudan University Shanghai Cancer Center

\section{Yiqun Cao}

Fudan University Shanghai Cancer Center

\section{Research Article}

Keywords: Glioblastoma multiforme, SPP1, PSMA, angiogenesis, HIF1a

Posted Date: April 6th, 2022

DOI: https://doi.org/10.21203/rs.3.rs-1266087/v2 
License: (c) (i) This work is licensed under a Creative Commons Attribution 4.0 International License. Read Full License 


\section{Abstract}

Background: Glioblastoma multiforme (GBM) is a highly vascular-rich and devastatingly aggressive type of malignant brain tumors, thus anti-angiogenesis therapy plays an essential role in the treatment of GBM. Our previous study had showed that the prostate-specific membrane antigen (PSMA) promoted angiogenesis of GBM through interacting with Integrin $\beta 4$ (ITGB4) and regulating NF-kB signaling pathway. This study aims to explore the upstream molecular mechanism of GBM regulating the expression of PSMA and promoting angiogenesis process.

Methods: Human cytokine antibody array and bioinformatics were used to explore potential cytokines involved in regulating PSMA expression. Recombinant protein was applied and western blot, qRT-PCR were assessed to verify that the contributing cytokine was SPP1. Potential transcription factor mediating the expression of PSMA was sifted through PROMO and JASPAR website. ChIP assay and dual luciferase were used to confirm that the transcription factor HIF-1a could regulate PSMA expression. Cellular migration and tube formation ability were assessed by scratch assay and tube formation assay in human umbilical vein endothelial cells (HUVECs). ELISA was conducted to evaluate the variation of SPP1 in the serum of GBM patients pre- and post-surgery. Expression levels, prognosis and receiver operation characteristic (ROC) curve of SPP1 were determined by bioinformatics to evaluate its clinical value.

Results: We demonstrated that cytokine SPP1 secreted from GBM could regulate the expression of PSMA in HUVEC. The mechanistic study revealed that SPP1 regulated the expression of PSMA through transcription factor HIF1a. Recombinant protein SPP1 could promote migration and tube formation ability of HUVEC, which could be blocked by knockdown of HIF1a. SPP1 was abundantly expressed in the GBM, and the high expression of SPP1 is associated with poor prognosis. Furthermore, SPP1 had a high value of clinical diagnosis with considerable sensitivity and specificity.

Conclusions: In this study, we identified that upstream cytokine SPP1 secreted from GBM could upregulate PSMA expression in endothelial cells via transcription factor HIF1a, which constructs a positive chain in angiogenesis and provides promising candidates for targeted therapy of GBM.

\section{Background}

Glioblastoma multiforme (GBM) is a highly invasive and devastatingly aggressive malignant brain tumor with an increasing incidence and a short median overall survival of about 16 months after diagnosis [1]. Despite a series of optimal treatments including radical surgical resection combined with standard radiotherapy and chemotherapy, the median survival of patients remains poor [2].

Unlike extracranial cancers, GBM infiltrates deeply into the surrounding brain parenchyma and hardly metastasizes out of the brain [3]. The extraordinary patterns of diffuse infiltration and recurrence are partly ascribed to tortuous blood vessels of GBM, which provide migration routes for tumor cells [4]. GBM is among the most highly vascularized tumors, due to the tumor-derived upregulation of angiogenic receptors and factors that stimulate angiogenesis signaling $[5,6]$. Hence, anti-angiogenesis therapies 
have attracted broad interest because of the correlation of angiogenesis with GBM prognosis and ease of exposure to targeted drugs [7-9]. However, since GBM is a highly heterogeneous and complex tumor with particularly invasive properties, most anti-angiogenesis therapies have hitherto exhibited limited efficacy in clinical trials $[10,11]$. Furthermore, most anti-angiogenesis therapies target vascular endothelial growth factor (VEGF), other effective molecular candidates are needed to explore further [5]. Hence, in-depth investigations of the molecular mechanism underlying GBM angiogenesis are conducive to identifying effective treatments.

Our previous study revealed that the prostate-specific membrane antigen (PSMA) plays a pivotal role in GBM angiogenesis [12]. PSMA, encoded by the gene folate hydrolase 1, is a type II transmembrane glycoprotein acting as glutamate carboxypeptidase on different substrates, including the nutrient folate and neuropeptide $\mathrm{N}$-acetyl-I-aspartyl-I-glutamate [13]. PSMA overexpression in endothelial cells has been associated with aggressiveness and rich neovasculature in various cancers [14-17]. In our previous study, we demonstrated that PSMA was robustly expressed in the vascular endothelial cells of GBM, and significantly associated with poor prognosis [12]. A series of in vitro and in vivo experiments demonstrated that PSMA overexpression facilitated endothelial cell proliferation, migration and tube formation in GBM via interactions with integrin $\beta 4$ (ITGB4) and activation of the nuclear factor (NF)-KB signaling pathway [12]. Therefore, PSMA may be of paramount importance in GBM angiogenesis and could been seen as a potential candidate for targeted therapy. Although we proved that PSMA is highly expressed in the neovasculature of GBM but not in normal brain blood vessels, the mechanism of PSMA expression regulation in GBM remained unknown.

Consequently, this study aimed to disclose the mechanism of PSMA upregulation in GBM. Previous studies showed that multiple angiogenic regulators could mediate GBM angiogenesis, such as VEGF, fibroblast growth factor (FGF), and angiopoietin-1 [6, 18-20]. In this study, we discovered that secreted phosphoprotein 1 (SPP1) is the GBM-secreted contributing factor that promotes PSMA upregulation in endothelial cells. This is in line with the findings of preceding studies showing that SPP1 displayed a beneficial effect on angiogenesis in diseases, such as atrophic fracture and colon cancer recurrence after therapy [21,22]. Furthermore, our study also showed that the expression of hypoxia inducible factor 1 alpha (HIF1a), a potent transcription factor that binds the PSMA promoter region, was positively regulated by SPP1. HIF1a, as a responsive factor to hypoxia, regulated the expression of many angiogenesis-related genes, thus playing an essential role in tumor angiogenesis [23-25]. Moreover, our study disclosed that SPP1 and HIF1a were both involved in the upregulation of PSMA, while knockdown of HIF1a reversed the SPP1-induced effects. Collectively, our data support that GBM-secreted SPP1 upregulated the expression of PSMA via transcription factor HIF1 a to facilitate GBM angiogenesis.

\section{Methods}

\section{Cell culture and preparation of conditioned medium}


Human umbilical vein endothelial cells (HUVECs), the human glioblastoma cell lines U87 and U251, and human microglial cell line HMC3 were acquired from the cell library of the Chinese Academy of Sciences. Cells were cultured in Dulbecco's modified Eagle's medium (DMEM) (Gibco) supplemented with 10\% fetal bovine serum (FBS, Gibco) and $1 \%$ penicillin-streptomycin reagent (Gibco), and incubated at $37^{\circ} \mathrm{C}$ in a humidified atmosphere with $5 \% \mathrm{CO} 2$. The medium was refreshed every two days. Controlled cell numbers of U87, U251, and HMC3 cells were cultured in serum-free medium for $24 \mathrm{~h}$. Then, the cell supernatant was collected as conditioned medium for use in the human cytokine antibody array.

\section{Human cytokine antibody array}

A human cytokine antibody array (RayBio ${ }^{\circledR}$ C-Series Human Cytokine Antibody Array C5, Ray Biotech, USA) including 80 different cytokines was used to measure the levels of several cytokines in the conditioned medium of U87, U251 and HMC3 cells, following the manufacturer's instructions. Next, we analyzed the 80 cytokines in the conditioned medium of U87, U251 glioma cells and HMC3 cells, and ranked the top 40 cytokines according to the $P$ value from small to large in the form of the heatmap. Among them, the most important cytokines with a significant difference $(P<0.05)$ were SPP1, TNFa, GCSF, ENA-78, NT-3, while the other cytokines had no significant difference. Analysis was performed using $\mathrm{R}$ and the limma package (an R package for differential analysis).

\section{RNA isolation and quantitative real-time PCR}

Total RNA was extracted using the TRIzol reagent (Invitrogen, Carlsbad, CA, USA). The RNA quality and quantity were evaluated as previously described [12]. Quantitative real-time PCR was also performed as previously described [12]. The expression levels of GAPDH, used as control, and targeted genes were assessed. All reactions were run in triplicate. Primer sequences were as follows:

h-GAPDH-F, 5-ACAACTTTGGTATCGTGGAAGG-3,

h-GAPDH-R, 5-GCCATCACGCCACAGTTTC-3;

h-PSMA-F, 5-ACACAGATACCACATTTAGCAGG-3,

h-PSMA-R, 5-TTTGGGTAGGACAACAGGACA-3;

h-HIF1a-F, 5-ATCCATGTGACCATGAGGAAATG-3,

h-HIF1a-R, 5-TCGGCTAGTTAGGGTACACTTC-3.

\section{Protein extraction and western blot analysis}


Cell pellets were washed twice in cold PBS (Gibco), and then lysed in RIPA buffer supplemented with 1\% PMSF and $1 \%$ phosphatase inhibitor on ice. The protein concentration was determined using a BCA protein assay kit (Beyotime Biotechnology).

Protein samples $(10 \mu \mathrm{g})$ were handled and transferred to membranes as previously described, then incubated with PSMA (1:1,000), HIF1a $(1: 1,000)$, and GAPDH $(1: 2,000)$ antibodies at $4{ }^{\circ} \mathrm{C}$ overnight [12]. Proteins were washed there times with TBST, and incubated with the corresponding secondary antibodies $(1: 5,000)$ at room temperature for $2 \mathrm{~h}$. The membranes were washed again, and then incubated with enhanced chemiluminescence (Thermo Fisher Scientific) for $1 \mathrm{~min}$. Finally, the protein band images were captured and analyzed.

\section{Promoter activity assessment using a dual-luciferase assay}

The Dual-Luciferase ${ }^{\circledR}$ Reporter Assay kit (Promega) was used to evaluate promoter activity following the manufacturer's instructions. The luciferase reporter construct PSMA-pGL3-Promoter-Luc was transiently co-transfected into HUVECs grown in 96-well plates using LipofectamineTM 3000 (Invitrogen). HUVECs were previously treated and grouped correspondingly (+/- HIF1a knockdown, +/-SPP1 or PBS, respectively). Both firefly and Renilla luciferase activities were analyzed at $72 \mathrm{~h}$ after infection using a dual-luciferase system on GloMax ${ }^{\circledR}$ Discover (Promega, Madison, USA).

\section{Chromatin immunoprecipitation (ChIP) assay}

For ChIP analysis, HUVECs were treated with $1 \mu \mathrm{g} / \mathrm{ml} \mathrm{SPP} 1$ or PBS and harvested separately. Cell samples in each dish with $8 \mathrm{ml}$ medium were fixed using $210 \mu \mathrm{l} 37 \%$ formaldehyde for $10 \mathrm{~min}$, and then the fixation was stopped by adding $400 \mu \mathrm{l} 2.5 \mathrm{M}$ glycine. The mix was slowly shaken for 2 min until the liquid turned yellow and then washed three times with precooled PBS. Next, $1 \mathrm{ml}$ PBS was added to each dish, the cells were scraped down into the EP tube, and centrifuged at $9000 \mathrm{rpm}$ for $30 \mathrm{~s}$. Finally, the cells were collected and supernatants discarded. The precipitate was lysed with $400 \mu \mathrm{l}$ 1\% SDS lysate, mixed and incubated on ice for $10 \mathrm{~min}$, followed by sonication on ice with $22 \%$ power and centrifugation at $13200 \mathrm{rpm}$ for $10 \mathrm{~min}$ at $4^{\circ} \mathrm{C}$. Finally, $300 \mu \mathrm{l}$ supernatant was collected. Electrophoresis was conducted to ensure the majority of the DNA fragments were between 300-700 bp and the rest of the supernatant was stored at $-80^{\circ} \mathrm{C}$. A total of $300 \mu \mathrm{l}$ supernatant was diluted with $0.6 \mathrm{~mL}$ dilution buffer containing PMSF (sigma). Agarose A or $\mathrm{G}$ was washed three times with TE. Each EP tube was prepared with $300 \mu \mathrm{l}$ chromatin, $1.2 \mathrm{ml}$ dilution buffer and $80 \mu \mathrm{l}$ beads (50\% turbidness), and then rotated for $1 \mathrm{~h}$ at $4^{\circ} \mathrm{C}$. After centrifugation at 4,000 rpm for $2 \mathrm{~min}, 50 \mu \mathrm{l}$ supernatant was obtained as input. The supernatants were divided into two portions ( $475 \mu \mathrm{l}$ each), one of which was incubated with rotation overnight in a cold chamber with the PMSA antibody $(2 \mu \mathrm{g})$ and the other with the same amount of normal lgG. The precipitated DNA was recovered using a PCR purification kit (TransGen Biotech) and analyzed using qRTPCR with a SYBR Green Real-Time PCR Master Mix (Fermentas). ChIP values were normalized to their 
respective input values, and the fold changes in concentration were assessed based on the relative enrichment in anti-PSMA compared with anti-IgG immunoprecipitates.

\section{Tube formation assay}

HUVECs with HIF1a knocked down or not were cultured under different treatment conditions, adding recombinant protein SPP1 $(1 \mu \mathrm{g} / \mathrm{ml})$ or PBS, in a 24-well plate pre-coated with Matrigel $(50 \mu \mathrm{l} /$ well, corning) for $24 \mathrm{~h}$. Capillary-like tube formation was photographed under an inverted microscope. Tube length and branching points were calculated using the NIH ImageJ software.

\section{Scratch assay}

HUVECs were seeded and cultured under different treatment conditions (+/- HIF1a knockdown, +/- SPP1 or PBS, respectively) in a 6 -well plate and grew to $100 \%$ confluency. Pipette tips of $200 \mu$ l were used to scratch the cell monolayer. Then, the plate was gently washed with PBS to clear detached cells. Images were captured at $0 \mathrm{~h}$ and $48 \mathrm{~h}$ under an inverted microscope.

\section{Clinical specimens}

Serum specimens were collected from normal human volunteers $(n=20)$, pre-operative GBM patients $(n=$ $20)$ and post-operative GBM patients within $72 h(n=20)$, and stored at $-80^{\circ} \mathrm{C}$. Patients $(n=20)$ received surgical treatment at Fudan University Shanghai Cancer Center between January 2021 and June 2021. Informed consents were collected from all patients and volunteers. Ethical approval was obtained from the ethics committee of the Fudan University Shanghai Cancer Center.

\section{Enzyme-linked immunosorbent assay (ELISA)}

ELISA was performed to assess the SPP1 levels in serum samples using a commercial kit according to the manufacturer's instructions (ELH-OPN-1; R\&D company, USA). Serum samples were diluted 25-fold, and then directly added to the specific 96-well plate previously coated with a human SPP1 antibody contained in the kit. The absorbance value was assessed at $450 \mathrm{~nm}$ using a microtest plate spectrophotometer. The SPP1 levels were quantified based on a standard SPP1 curve.

\section{Bioinformatic analysis}

The clinical analysis of SPP1, including expression levels, the Kaplan-Meier curves of overall survival and the receiver operator characteristics (ROC) curve, was performed using The Cancer Genome Atlas (TCGA) database datasets. Moreover, the high and low SPP1 expression groups were divided by SPP1 expression 
mean. The correlations of targeted genes were assessed using GEPIA (http://gepia.cancer-pku.cn/). The open-access transcription factor database JASPAR (http://jaspar.genereg.net) was used to explore potential transcription factors binding to PSMA promotor.

\section{Statistical analysis}

Data are presented as the mean \pm SD. The data were analyzed using Graphpad Prism 9.0 software, and independent Student's tests (two-tailed) and one-way ANOVA tests were used to analyze the differences between groups. Pearson and Spearman correlation analysis was used for correlation analysis. $P$ value $<$ 0.05 was considered statistically significant.

\section{Results}

\section{Screening cytokines secreted from glioma cells}

We previously demonstrated that HUVECs cultured with conditioned medium from U87 and U251 glioma cells exhibited significantly higher PSMA expression than that of cells cultured with normal medium [12]. To identify the factor in the conditioned medium from U87 and U251 cells that affected PSMA expression in HUVECs, we used a Human Cytokine Antibody Array to screen different cytokines in the conditioned medium from U87 and U251 cells relative to those of HMC3 cells (Fig. 1A) and identified 39 upregulated and five downregulated cytokines in the conditioned medium from U87 and U251 cells (Fig. 1B). A heatmap was constructed to exhibit the most significant differential cytokine levels between those in the conditioned medium from U87 and U251 cells and those of HMC3 cells (Fig. 1C). The volcano plot demonstrated four upregulated cytokines (SPP1, G-CSF, NT-3, TNFa) and one downregulated cytokine (ENA-78/CXCL5) with the most marked difference (Fig. 1D).

\section{SPP1 from glioma cells regulates PSMA expression}

To identify a more precise target, we analyzed the correlation of PSMA with cytokines (SPP1, G-CSF, TNFa, NT-3, ENA-78) in GBM using GEPIA. Bioinformatic analyses showed that SPP1 exhibited significant correlation with PSMA $(\mathrm{R}=0.28, P<0.01)$ (Fig. 2A). Correlations of other cytokines with PSMA were not as marked as that with SPP1 whether they were upregulated or downregulated in the conditioned medium (Fig. 2A\&B). Previous reports also showed that SPP1 was associated with angiogenesis in other cancers, such as colon cancer and melanoma [26,27]. Hence, we extrapolated that SPP1 might be the contributing factor. We added recombinant protein SPP1 $(1 \mu \mathrm{g} / \mathrm{ml})$ into the culture medium of HUVECs and PBS as control. Accordingly, the mRNA and protein expression levels of PSMA were assessed using qRT-PCR and western blot. We found that secreted SPP1 protein had a significant effect on PSMA upregulation in HUVECs (Fig. 2C\&D). Hence, we confirmed that glioma-secreted cytokine SPP1 was the contributing factor that positively upregulated PSMA in HUVECs. 


\section{SPP1 promotes PSMA upregulation through transcription factor HIF1a}

To reveal the mechanism underlying SPP1 regulation of PSMA expression, we sifted through the PROMO website to identify potential transcription factors that predominantly bind to the PSMA promoter region. We found that HIF1 a could be a potent transcription factor with highly conserved binding sites in the upstream of the PSMA promoter region using JASPAR $(P<0.01)$ (Fig. 3A\&B). Hence, we extrapolated that SPP1 might upregulate the expression of PSMA through regulating HIF1a.

To verify our speculation, we knocked down HIF1a in HUVECs using siRNA and examined the knockdown efficiency using qRT-PCR $(P<0.01)$ (Fig. 3C). Furthermore, we manipulated the concentration level of SPP1 by adding recombinant protein. Western blot and qRT-PCR results showed that HIF1a knockdown mitigated the expression of PSMA $(P<0.05)$ (Fig. 3D\&E). It also confirmed that SPP1 upregulated the expression of PSMA $(P<0.01)$, which could be reversed by knockdown of HIF1a $(P<0.01)$ (Fig. 3D\&E). Intriguingly, the western blot results showed that SPP1 alone could upregulate the expression of HIF1a, irrespective of whether HIF1a was knocked down or not $(P<0.05)$ (Fig. 3D). Thus, our results confirmed that SPP1 could upregulate the expression of PSMA through enhancing HIF1a expression.

To further verify that HIF1a could truly occupy binding sites of PSMA promotor, we designed primers covering the three binding sites of PSMA promoter and conducted ChIP assays. The results showed that HIF1a bound region 1 of the PSMA promoter (h-PSMA-promoter-F1, CAAATGCACGGCCTCTCTCA; $h$ PSMA-promoter-R1, TATCCCGGCTATGTCTGGCT), which was significantly enhanced in the presence of SPP1 $(P<0.01)$ (Fig. 3F). We used a Dual-Luciferase Report gene assay to further assess the impact of HIF1 $a$ and SPP1 on the transcriptional activity of the PSMA promoter in HUVECs. We found that knockdown of HIF1a alone undermined the relative luciferase activity, which was correlated with the transcriptional level of the PSMA-pGL3-promoter, while using recombinant protein SPP1 alone had the opposite effect $(P<0.05)$ (Fig. 3G). The combination of downregulated HIF1a and recombinant protein SPP1 had a neutralizing effect $(P<0.05)$ (Fig. 3G). Altogether, the Dual-Luciferase report gene assay demonstrated that SPP1 and HIF1a both had a positive influence on the transcriptional process of the PSMA promoter, also indicating the affinity of HIF1a for the PSMA promoter.

Our findings strongly support that SPP1 upregulates the expression of PSMA through increasing the expression of HIF1a, which has a conservative binding site on the PSMA promoter region.

\section{The ability of SPP1-regulated endothelial migration and tube formation could be blocked by knocking down HIF1a}

We previously demonstrated that PSMA overexpression affected biological functions, such as HUVEC migration and tube formation in vivo and in vitro[12]. As mentioned above, we confirmed that GBMsecreted SPP1 could upregulate PSMA expression in HUVECs through transcription factor HIF1a. 
Therefore, we hypothesized that SPP1 could also promote migration and tube formation ability of HUVECs and it could be inhibited by knockdown of HIF1a. To verify our speculation, we added recombinant protein SPP1 $(1 \mu \mathrm{g} / \mathrm{ml})$ or the same volume of PBS into the culture medium of HUVECs with HIF1 a knockdown or not. The scratch assay showed that SPP1 promoted HUVEC migration while downregulating HIF1a expression significantly reversed the effect $(P<0.01)$ (Fig. 4A\&C). In parallel, the tube formation assay showed that SPP1 significantly enhanced HUVEC tube formation. The ability of SPP1-regulated endothelial migration and tube formation could be blocked by knocking down HIF1a $(P<$ 0.05) (Fig. 4B\&D). Collectively, all the above results confirmed our previous speculation that GBMreleased SPP1 could promote migration and tube formation of surrounding vascular endothelial cells, which would result in GBM angiogenesis and progression.

\section{SPP1 is abundantly expressed in GBM and predicts poor prognosis}

To explore its expression and diagnostic value in GBM, we then analyzed the expression levels and prognosis of SPP1 in GBM using TCGA datasets. The results showed that SPP1 expressed higher compared with that in the normal tissues both in LGG and GBM group $(P<0.01)$ (Fig. 5A). Furthermore, Kaplan-Meier curves of overall survival indicated that high expression of SPP1 was associated with poor prognosis both in LGG and GBM group $(P<0.01)$ (Fig. 5B). To verify the bioinformatic results, we conducted ELISA to assess the concentration level of SPP1 in serum of GBM patients before and after surgery compared with normal people. Compared with normal people, the expression of SPP1 in preoperative GBM patients was higher. However, the expression levels of SPP1 became lower when the GBM tumor was resection $(P<0.01)$ (Fig. $5 C)$. The ELISA results indicated that SPP1 might be a potential diagnostic and curative biomarker in GBM patients. To determine whether SPP1 could act as a promising marker, the ROC curve was also constructed using TCGA datasets. The ROC curve demonstrated that SPP1 scores had an AUC of 0.785 (95\% confidence interval $(\mathrm{Cl})=0.762-0.807)$ in LGG group, and an even higher AUC of $0.957(95 \% \mathrm{Cl}=0.944-0.971)$ in GBM group, indicating that SPP1 could be a potent diagnostic molecule in GBM with considerable sensitivity and specificity for future clinical application (Fig. 5D). Altogether, our results showed that SPP1 was abundantly expressed and could predict poor prognosis in GBM, indicating its high potential as a promising target for future diagnosis with considerable sensitivity and specificity.

\section{Discussion}

Accumulating evidence demonstrated that PSMA was highly expressed in the neovasculature of various cancers, including GBM $[14-17,28]$. Most studies focused on using PET/CT loaded with Ga-PSMA-11 or anti-PSMA minibody as an efficient imaging tool. Other studies elucidated that PSMA was highly expressed in the microvascular of many tumors [28-31]. Our previous study demonstrated that PSMA was highly expressed in vascular endothelial cells in GBM and that it facilitated angiogenesis through interacting with ITGB4 and stimulating the NF-KB signaling pathway [12]. However, the upstream 
molecular mechanism of GBM regulating the expression of PSMA and promoting angiogenesis process remains unclear.

It was merely reported that PSMA expression was regulated by a cis-element, the PSMA enhancer (PSME), in prostate epithelium [32]. Moreover, PSME could be negatively regulated by Sox7 protein [33]. As the above mechanism was not exclusive to tumors, it could not apply to our study, which unraveled the specific mechanism of GBM-induced PSMA upregulation. Our previous study demonstrated that conditioned medium from glioma cells could induce PSMA upregulation in HUVECs. Therefore, our present study tried to explore mechanism of GBM regulating the expression of PSMA in conditioned medium from glioma cells. Supported by bioinformatic analysis and experimental verification, we confirmed that SPP1 was the pivotal GBM-derived factor that could regulate PSMA expression. In addition, both the number of cells treated with SPP1 and the duration of treatment were able to affect the degree of upregulation of PSMA expression, as seen in different elevated extent of PSMA expression in qRT-PCR and luciferase assay. Furthermore, SPP1 could up-regulated the expression of PSMA and promote the ability of migration and tube formation of HUVECS. To the best of our knowledge, this study is the first to reveal the relationship between SPP1 and PSMA.

Our study focused on the role of SPP1 in tumor angiogenesis, while in fact, SPP1 has a broad biological role in cancers, indicating its promising value in clinical diagnosis and therapy. SPP1, which has different isoforms from several transcript variants, is a secreted protein involved in osteoclast attachment to the mineralized bone matrix [34]. SPP1 also upregulates the expression of interferon- $y$ and interleukin-12, and is involved in Th1-mediated immunity $[35,36]$. Many studies revealed that SPP1 is involved in angiogenesis in tumors like breast cancer, lung cancer, melanoma and colon cancer [26, 27, 37-39]. Despite the lack of mechanistic research, SPP1 has been highly associated with GBM angiogenesis using IHC, which consolidated our initial conclusion $[40,41]$. Notably, SPP1 positively upregulated VEGF expression, which could be a potential mechanism of SPP1-promoted angiogenesis [40, 42]. Intriguingly, PMSA-stimulated NF-kB activation was required for VEGF expression [43]. Hence, there might be a potential link between SPP1, PSMA and VEGF, which requires in-depth investigation in the future to broaden our understanding of GBM angiogenesis. Besides angiogenesis, SPP1 plays a supportive role in tumor progression processes, such as proliferation, invasion, migration and resistance to chemotherapy in multiple cancers [44-47]. Moreover, SPP1 is involved in creating the immunosuppressed tumor microenvironment $[48,49]$. The abovementioned studies indicated that SPP1 might also participate in various processes of GBM progression beyond angiogenesis, inspiring us to conduct a comprehensive study of SPP1 in GBM in the future.

SPP1 is highly expressed in multiple cancers, including lung cancers, head and neck cancers, liver cancer, colon adenocarcinoma, melanoma and GBM, and correlates with poor prognosis $[44,45,48-50]$. We demonstrated that SPP1 was abundantly expressed in GBM compared with its levels in normal tissues, and positively associated with poor prognosis. Interestingly, the concentration of SPP1 in the peripheral blood of GBM robustly mitigated after surgery compared with that before surgery, further corroborating the significant clinical correlation of SPP1 with GBM. Importantly, our study is the first to assess the 
expression level of SPP1 in peripheral serum of GBM patient, since preceding studies focused on SPP1 expression in glioma cell lines or tissues from surgical resection using IHC and western blot [51]. Furthermore, SPP1 had a high diagnostic value in GBM with considerable sensitivity and specificity. In conclusion, our result indicated that serum SPP1 combined with PSMA-PET/CT is of great significance in clinical diagnosis of GBM progression and recurrence in the future.

Following a series of rigorous evidence, we proved that HIF1a was the pivotal link between SPP1 and PSMA. HIF1a was mediated by SPP1 and acted as a potent transcription factor on the binding site of the PSMA promoter. HIF1a is famous for its response to hypoxic environments to maintain homeostasis, mediating various cellular biological states, such as metabolism, inflammation and angiogenesis [2325]. The involvement of HIF1a in angiogenesis has long been reported. Because cells in hypoxic microenvironments are striving for more oxygen and nutrients, newly sprouted vessels are needed as a pathological response to hypoxia [5]. Thus, our study provides a greatly promising candidate for future targeted GBM diagnosis and therapy.

GBM has the diffuse infiltration and heterogeneous pattern, which makes it more recurrence even though resection followed by radiotherapy. Compared with traditional treatment, targeting the abnormal GBM angiogenesis seems to be a more promising therapy. However, a few limitations of our study remain to be addressed, such as the limited number of clinical specimens. Also, our experiment has only initially confirmed the correlation between SPP1 and PSMA, while the changes of PSMA expression under different doses of SPP1 treatment have not yet been explored, which could help verify if a dosedependent link does exist.

\section{Conclusions}

In summary, we identified that upstream cytokine SPP1 secreted from GBM could upregulate PSMA expression in endothelial cells via transcription factor HIF1a, which constructs a positive chain in angiogenesis and provides promising candidates for targeted therapy of GBM (Fig. 6).

\section{Abbreviations}

SPP1: Secreted Phosphoprotein 1; GBM: Glioblastoma multiforme; PSMA: prostate-specific membrane antigen; HUVECs: human umbilical vein endothelial cells; VEGF: vascular endothelial growth factor; FOLH1: Folate Hydrolase 1; ITGB4: Integrin $\beta 4$; FGF: fibroblast growth factor; HIF1a: Hypoxia Inducible Factor 1 Subunit Alpha; ELISA: enzyme-linked immunosorbent assay; ChIP: Chromatin immunoprecipitation; LGG: low grade glioma; ROC: receiver operator characteristics; Cl: confidence interval; IHC: Immunohistochemistry.

\section{Declarations}

\section{Acknowledgments}


We thank the editors at Elsevier Language Editing Services for their help in editing our manuscript.

\section{Authors' contributions}

YG and WJT conceived the concept and designed the experiments; LDL, CSZ and MTF performed all experiments; LC and DHL conducted the data collection; WJT and HZ contributed to original manuscript drafting, which was checked by $Y G$ and $Y Q C ; X C, B H$ contributed to manuscript editing. All authors read and approved the final manuscript.

\section{Funding}

This work was supported by the National Natural Science Foundation of China (No. 882103429 and 82173177), the Foundation of Tenth People's Hospital (No. 04.03.18.088), the Foundation of Shanghai Municipal Health Bureau (No. 20204Y0262).

\section{Availability of data and materials}

All data generated or analyzed during this study are included in this published article (and its supplementary files). Our data of bioinformatic analysis are from GEPIA (http://gepia.cancer-pku.cn/) and Xiantao bioinformatic website (https://www.xiantao.love/databases, accession numbers is 15821960178).

\section{Declarations}

\section{Ethics approval and consent to participate}

The study involving human serum samples was approved by the ethics committee of the Fudan University Shanghai Cancer Center and all patients were provided informed consent for participation. We confirmed that all experiments were performed in accordance with relevant guidelines and regulations.

\section{Consent for publication}

Not applicable.

\section{Competing interests}

The authors declare that the research was conducted in the absence of any commercial or financial relationships that could be construed as a potential conflict of interest. 


\section{Authors' information}

${ }^{1}$ Department of Neurosurgery, Fudan University Shanghai Cancer Center, Shanghai, China. ${ }^{2}$ Department of Oncology, Shanghai Medical College, Fudan University, Shanghai, China. ${ }^{3}$ Department of Nuclear Medicine, Shanghai Tenth People's Hospital, Tongji University, Shanghai, China

\section{References}

1. Vollmann-Zwerenz A, Leidgens V, Feliciello G, Klein CA, Hau P. Tumor cell invasion in glioblastoma. Int J Mol Sci. 2020;21:1-21.

2. Khaddour K, Johanns TM, Ansstas G. The landscape of novel therapeutics and challenges in glioblastoma multiforme: Contemporary state and future directions. Pharmaceuticals. 2020;13:1-26.

3. Li C, Wang S, Yan JL, Torheim T, Boonzaier NR, Sinha R, et al. Characterizing tumor invasiveness of glioblastoma using multiparametric magnetic resonance imaging. J Neurosurg. 2020;132:1465-72.

4. Cuddapah VA, Robel S, Watkins S, Sontheimer H. A neurocentric perspective on glioma invasion. Nat Rev Neurosci. 2014;15:455-65.

5. Ahir BK, Engelhard HH, Lakka SS. Tumor Development and Angiogenesis in Adult Brain Tumor: Glioblastoma. Mol Neurobiol. 2020;57:2461-78.

6. Takano S, Yamashita T, Ohneda O. Molecular therapeutic targets for glioma angiogenesis. J Oncol. 2010;2010 Figure 4.

7. Wang Z, Yang Z, Jiang J, Shi Z, Mao Y, Qin N, et al. Silk Microneedle Patch Capable of On-Demand Multidrug Delivery to the Brain for Glioblastoma Treatment. Adv Mater. 2021;2106606:2106606.

8. Sousa F, Costa-Pereira Al, Cruz A, Ferreira FJ, Gouveia M, Bessa J, et al. Intratumoral VEGF nanotrapper reduces gliobastoma vascularization and tumor cell mass. J Control Release. 2021;339 September:381-90.

9. Frisch A, Kälin S, Monk R, Radke J, Heppner FL, Kälin RE. Apelin controls angiogenesis-dependent glioblastoma growth. Int J Mol Sci. 2020;21:1-19.

10. Parker JJ, Canoll P, Niswander L, Kleinschmidt-DeMasters BK, Foshay K, Waziri A. Intratumoral heterogeneity of endogenous tumor cell invasive behavior in human glioblastoma. Sci Rep. 2018;8:1-10.

11. Ameratunga $M$, Pavlakis $N$, Wheeler $H$, Grant $R$, Simes J KM. Anti-angiogenic therapy for high-grade glioma. Cochrane Database Syst Rev. 2018;11:CD008218.

12. Gao Y, Zheng H, Li L, Feng M, Chen X, Hao B, et al. Prostate-Specific Membrane Antigen (PSMA) Promotes Angiogenesis of Glioblastoma Through Interacting With ITGB4 and Regulating NF-KB Signaling Pathway. Front Cell Dev Biol. 2021;9 March:1-11.

13. Hyväkkä A, Virtanen V, Kemppainen J, Grönroos TJ, Minn H, Sundvall M. More than meets the eye: Scientific rationale behind molecular imaging and therapeutic targeting of prostate-specific membrane antigen (psma) in metastatic prostate cancer and beyond. Cancers (Basel). 2021;13. 
14. Chang SS, Reuter VE, Heston WDW, Bander NH, Grauer LS, Gaudin PB. Five different anti-prostatespecific membrane antigen (PSMA) antibodies confirm PSMA expression in tumor-associated neovasculature. Cancer Res. 1999;59:3192-8.

15. Conway RE, Rojas C, Alt J, Nováková Z, Richardson SM, Rodrick TC, et al. Prostate-specific membrane antigen (PSMA)-mediated laminin proteolysis generates a pro-angiogenic peptide. Angiogenesis. 2016;19:487-500.

16. Wernicke AG, Varma S, Greenwood EA, Christos PJ, Chao KSC, Liu H, et al. Prostate-specific membrane antigen expression in tumor-associated vasculature of breast cancers. Apmis. 2014;122:482-9.

17. Watanabe R, Maekawa M, Kiyoi T, Kurata M, Miura N, Kikugawa T, et al. PSMA-positive membranes secreted from prostate cancer cells have potency to transform vascular endothelial cells into an angiogenic state. Prostate. 2021;81:1390-401.

18. Stacker SA, Achen MG. Emerging roles for VEGF-D in human disease. Biomolecules. 2018;8:1-17.

19. Lieu C, Heymach J, Overman M, Tran H, Kopetz S. Beyond VEGF: Inhibition of the fibroblast growth factor pathway and antiangiogenesis. Clin Cancer Res. 2011;17:6130-9.

20. Machein MR, Knedla A, Knoth R, Wagner S, NeuschI E, Plate KH. Angiopoietin-1 promotes tumor angiogenesis in a rat glioma model. Am J Pathol. 2004;165:1557-70.

21. Wang C, Ying J, Nie X, Zhou T, Xiao D, Swarnkar G, et al. Targeting angiogenesis for fracture nonunion treatment in inflammatory disease. Bone Res. 2021;9.

22. Chang J, Bhasin SS, Bielenberg DR et al. Chemotherapy-generated cell debris stimulates colon carcinoma tumor growth via osteopontin. FASEB J. 2019;33:114-25.

23. Blatchley MR, Hall F, Wang S, Pruitt HC, Gerecht S. Hypoxia and matrix viscoelasticity sequentially regulate endothelial progenitor cluster-based vasculogenesis. Trans Annu Meet Soc Biomater Annu Int Biomater Symp. 2019;40 March:649.

24. Masoud GN, Li W. HIF-1 a pathway: Role, regulation and intervention for cancer therapy. Acta Pharm Sin B. 2015;5:378-89.

25. Fu C, An N, Liu J, Jun A, Zhang B, Liu M, et al. The transcription factor ZFHX3 is crucial for the angiogenic function of hypoxia-inducible factor $1 \mathrm{a}$ in liver cancer cells. J Biol Chem. 2020;295:7060-74.

26. Wu XL, Lin KJ, Bai AP, Wang WX, Meng XK, Su XL, et al. Osteopontin knockdown suppresses the growth and angiogenesis of colon cancer cells. World J Gastroenterol. 2014;20:10440-8.

27. Kale S, Raja R, Thorat D, Soundararajan G, Patil T V., Kundu GC. Osteopontin signaling upregulates cyclooxygenase-2 expression in tumor-associated macrophages leading to enhanced angiogenesis and melanoma growth via a9ß1 integrin. Oncogene. 2014;33:2295-306.

28. Sasikumar A, Kashyap R, Joy A, Charan Patro K, Bhattacharya P, Reddy Pilaka VK, et al. Utility of 68Ga-PSMA-11 PET/CT in imaging of glioma - A pilot study. Clin Nucl Med. 2018;43:e304-9. 
29. Nomura N, Pastorino S, Jiang P, Lambert G, Crawford JR, Gymnopoulos M, et al. Prostate specific membrane antigen (PSMA) expression in primary gliomas and breast cancer brain metastases. Cancer Cell Int. 2014;14.

30. Matsuda M, Ishikawa E, Yamamoto T, Hatano K, Joraku A, lizumi Y, et al. Potential use of prostate specific membrane antigen (PSMA) for detecting the tumor neovasculature of brain tumors by PET imaging with 89 Zr-Df-IAB2M anti-PSMA minibody. J Neurooncol. 2018;138:581-9.

31. Kunikowska J, Bartosz K, Leszek K. Glioblastoma multiforme: another potential application for 68GaPSMA PET/CT as a guide for targeted therapy. Eur J Nucl Med Mol Imaging. 2018;45:886-7.

32. Watt F, Martorana A, Brookes DE, Ho T, Kingsley E, O'Keefe DS, et al. A tissue-specific enhancer of the prostate-specific membrane antigen gene, FOLH1. Genomics. 2001;73:243-54.

33. Peng W, Guo L, Tang R, Liu X, Jin R, Dong JT, et al. Sox7 negatively regulates prostate-specific membrane antigen (PSMA) expression through PSMA-enhancer. Prostate. 2019;79:370-8.

34. Denhardt DT, Noda M. Osteopontin expression and function: Role in bone remodeling. J Cell Biochem. 1998;102 SUPPL. 30/31:92-102.

35. Maeno Y, Nakazawa S, Dao LD, Van Tuan N, Giang ND, Van Hanh T, et al. Osteopontin is involved in Th1-mediated immunity against Plasmodium falciparum infection in a holoendemic malaria region in Vietnam. Acta Trop. 2006;98:305-10.

36. Renkl AC, Wussler J, Ahrens T, Thoma K, Kon S, Uede T, et al. Osteopontin functionally activates dendritic cells and induces their differentiation toward a Th1-polarizing phenotype. Blood. 2005;106:946-55.

37. Krstic M, Hassan HM, Kolendowski B, Hague MN, Anborgh PH, Postenka CO, et al. Isoform-specific promotion of breast cancer tumorigenicity by TBX3 involves induction of angiogenesis. Lab Investig. 2020;100:400-13.

38. Raineri D, Dianzani C, Cappellano G, Maione F, Baldanzi G, lacobucci I, et al. Osteopontin binds ICOSL promoting tumor metastasis. Commun Biol. 2020;3.

39. Giopanou I, Kanellakis NI, Giannou AD, Lilis I, Marazioti A, Spella M, et al. Osteopontin drives KRASmutant lung adenocarcinoma. Carcinogenesis. 2020;41:1134-44.

40. Takano S, Tsuboi K, Tomono Y, Mitsui Y, Nose T. Tissue factor, osteopontin, a(v) $\beta 3$ integrin expression in microvasculature of gliomas associated with vascular endothelial growth factor expression. Br J Cancer. 2000;82:1967-73.

41. Szulzewsky F, Schwendinger N, Güneykaya D, Cimino PJ, Hambardzumyan D, Synowitz M, et al. Loss of host-derived osteopontin creates a glioblastoma-promoting microenvironment. Neuro Oncol. 2018;20:355-66.

42. Lou H, Wu LQ, Wang H, Wei RL, Cheng JW. The potential role of osteopontin in the pathogenesis of Graves' ophthalmopathy. Investig Ophthalmol Vis Sci. 2021;62.

43. Liu M, Xu W, Su M, Fan P. REC8 suppresses tumor angiogenesis by inhibition of NF-kB-mediated vascular endothelial growth factor expression in gastric cancer cells. Biol Res. 2020;53:1-8. 
44. Guo Z, Huang J, Wang Y, Liu XP, Li W, Yao J, et al. Analysis of Expression and Its Clinical Significance of the Secreted Phosphoprotein 1 in Lung Adenocarcinoma. Front Genet. 2020;11 June:1-11.

45. Wang J, Hao F, Fei X, Chen Y. SPP1 functions as an enhancer of cell growth in hepatocellular carcinoma targeted by miR-181c. Am J Transl Res. 2019;11:6924-37.

46. Tang H, Chen J, Han X, Feng Y, Wang F. Upregulation of SPP1 Is a Marker for Poor Lung Cancer Prognosis and Contributes to Cancer Progression and Cisplatin Resistance. Front Cell Dev Biol. 2021;9 April:1-9.

47. Pang X, Zhang J, He X, Gu Y, Qian B, Xie R, et al. SPP1 Promotes Enzalutamide Resistance and Epithelial-Mesenchymal-Transition Activation in Castration-Resistant Prostate Cancer via PI3K/AKT and ERK1/2 Pathways. Oxid Med Cell Longev. 2021;2021:1-15.

48. Wei T, Bi G, Bian Y, Ruan S, Yuan G, Xie H, et al. The Significance of Secreted Phosphoprotein 1 in Multiple Human Cancers. Front Mol Biosci. 2020;7 November:1-17.

49. Deng G, Zeng F, Su J, Zhao S, Hu R, Zhu W, et al. BET inhibitor suppresses melanoma progression via the noncanonical NF-kB/SPP1 pathway. Theranostics. 2020;10:11428-43.

50. Chiu TJ, Lu HI, Chen CH, Huang WT, Wang YM, Lin WC, et al. Osteopontin Expression Is Associated with the Poor Prognosis in Patients with Locally Advanced Esophageal Squamous Cell Carcinoma Receiving Preoperative Chemoradiotherapy. Biomed Res Int. 2018;2018.

51. Chen J, Hou C, Zheng Z, Lin H, Lv G, Zhou D. Identification of Secreted Phosphoprotein 1 (SPP1) as a Prognostic Factor in Lower-Grade Gliomas. World Neurosurg. 2019;130:e775-85.

\section{Figures}


A.
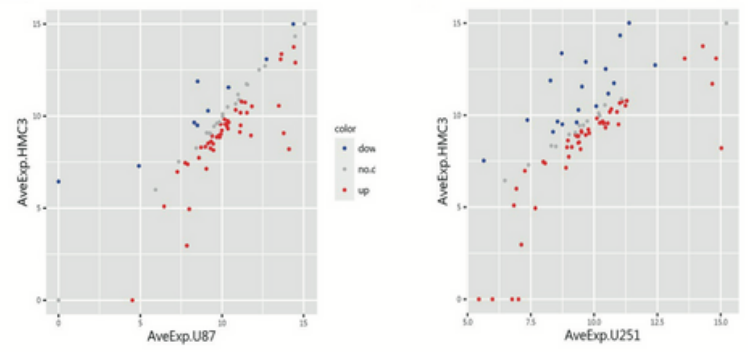

B.

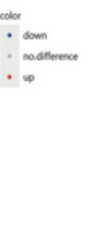

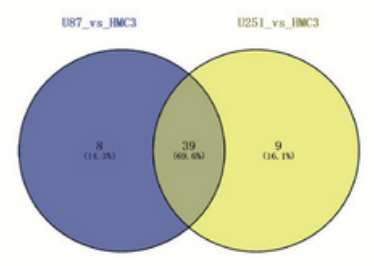

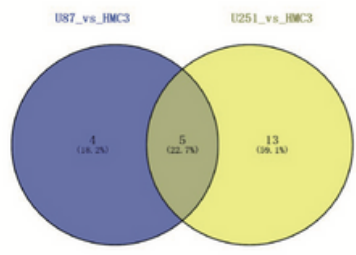

C.

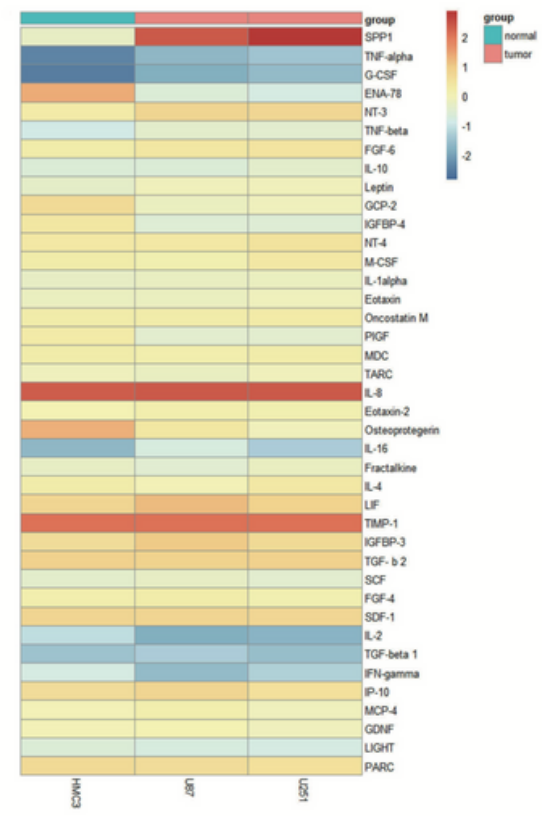

D.

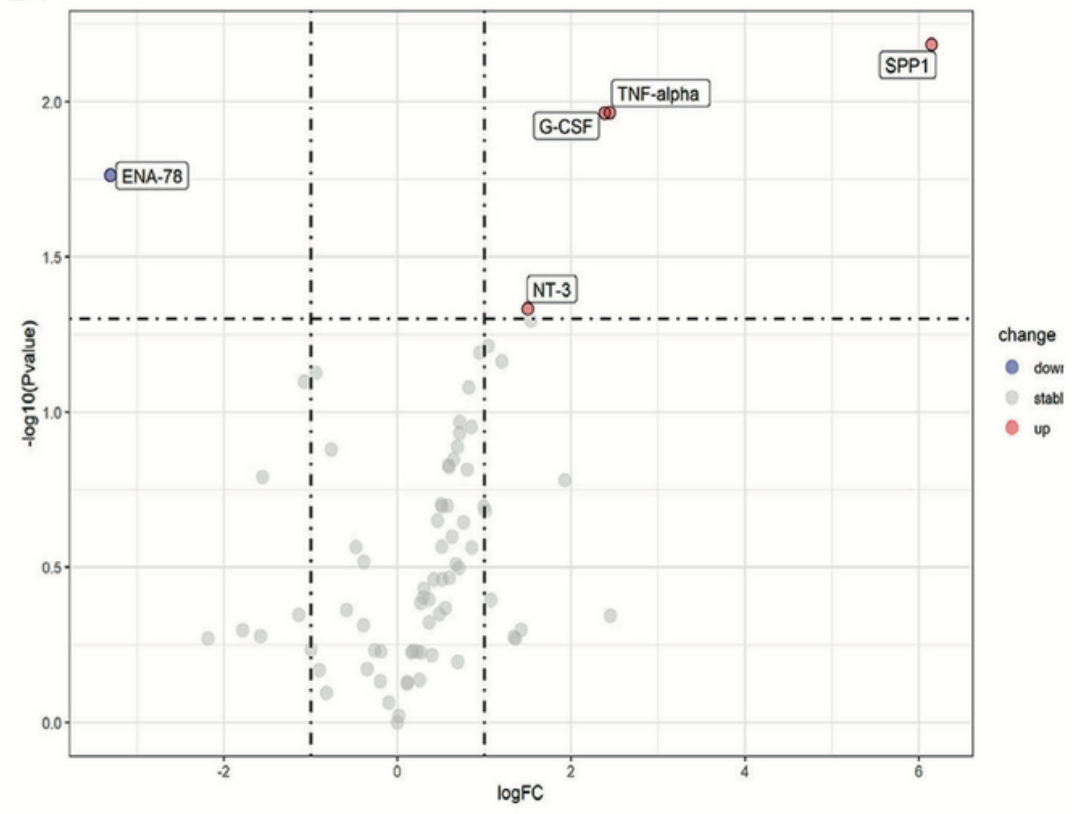

Figure 1

Screening cytokines secreted from glioma cells. A Human Cytokine Antibody Array of conditioned medium from U87, U251 and HMC3 cells; B Analysis of upregulated 39 cytokines and downregulated five cytokines in conditioned medium from U87 and U251 cells; C Heatmap analysis of the significant differential cytokines; D Volcano plot analysis of four marked up-regulated cytokines (SPP1, G-CSF, NT-3, TNFa) and one down-regulated cytokine (ENA-78). 
A.
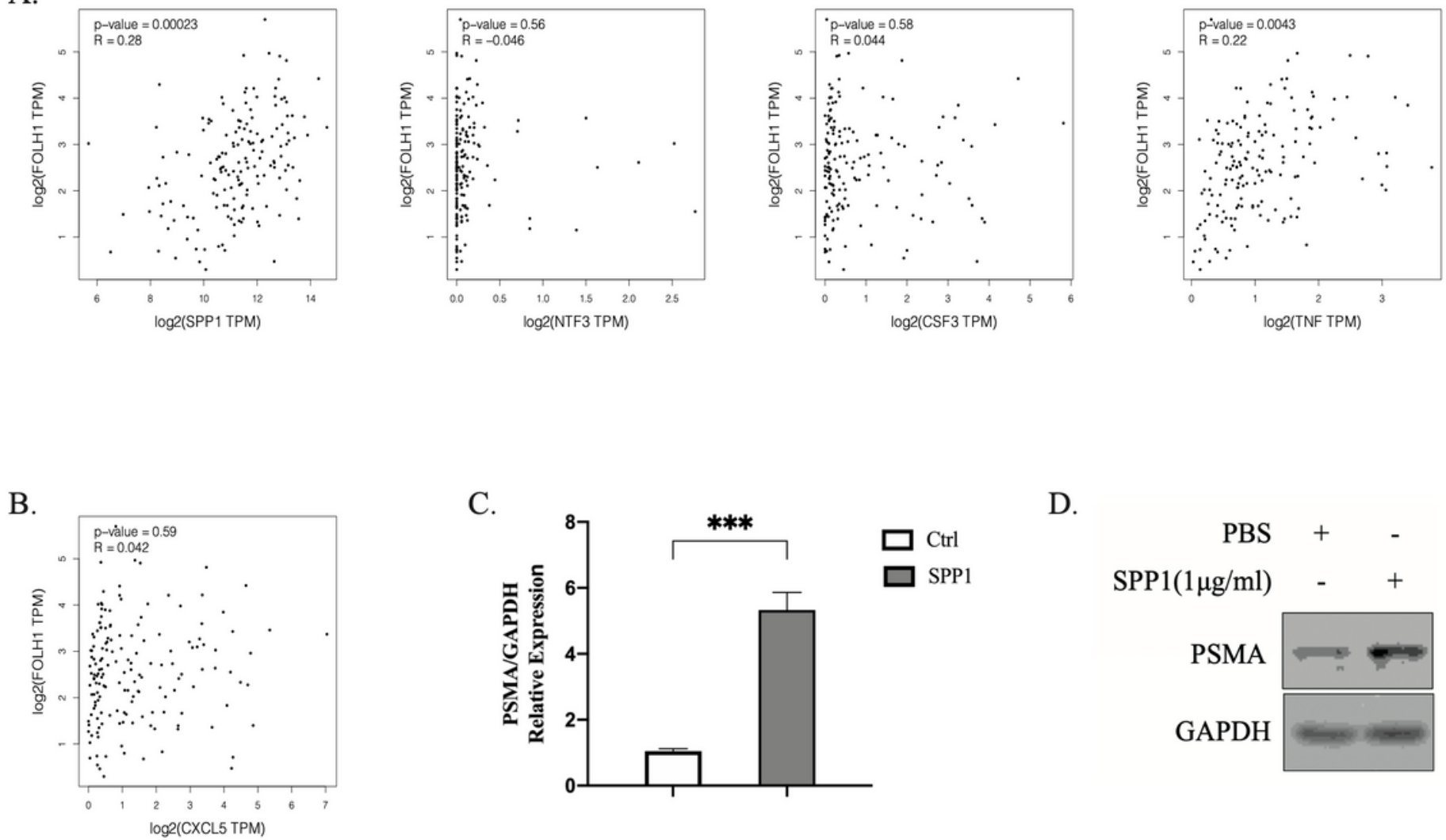

C.

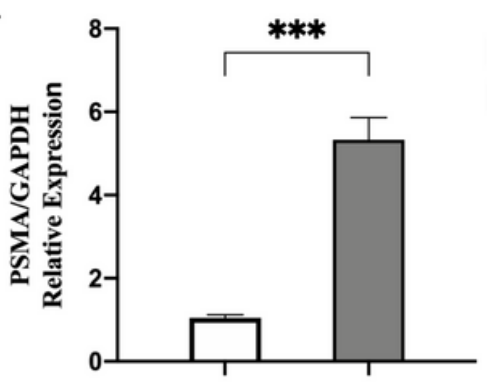

D.

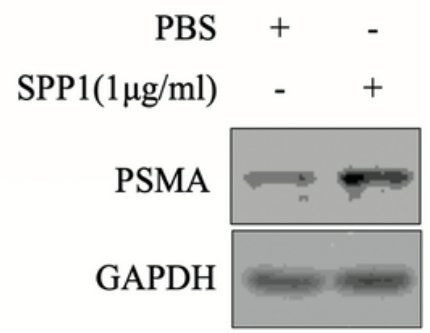

Figure 2

SPP1 from glioma cells regulates PSMA expression. A The correlation analysis between PSMA and upregulated cytokines (SPP1, NT-3/NTF3, G-CSF/CSF3 and TNFa/TNF) in GBM by GEPIA; B The correlation analysis between PSMA and downregulated cytokine ENA-78/CXCL5 in GBM by GEPIA; C-D The expression of PSMA after treatment with recombinant protein SPP1. 
A.

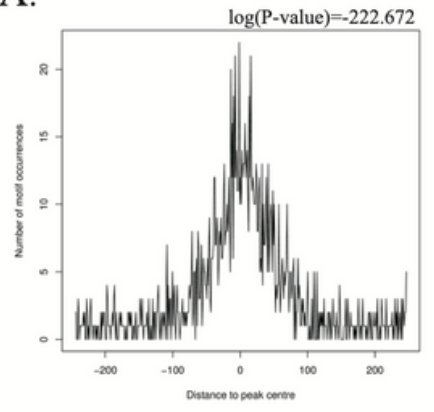

C.

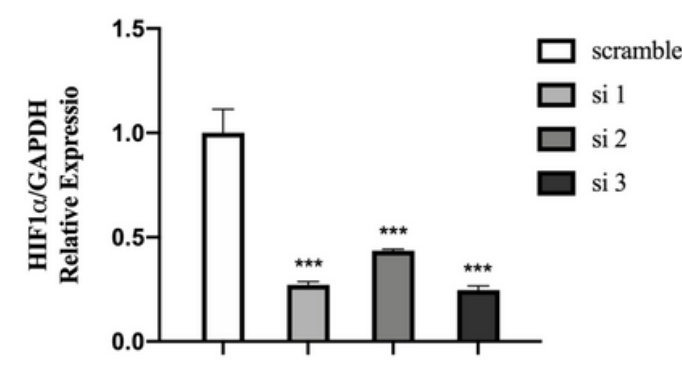

F.

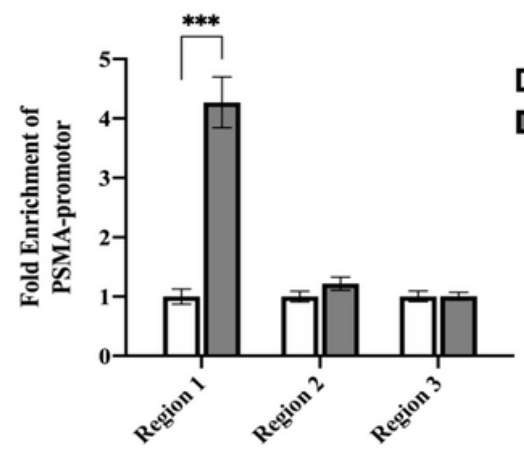

B.

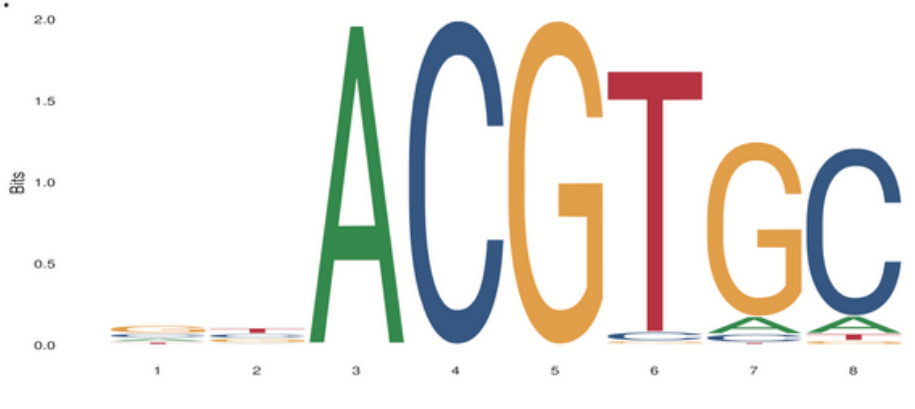

D.

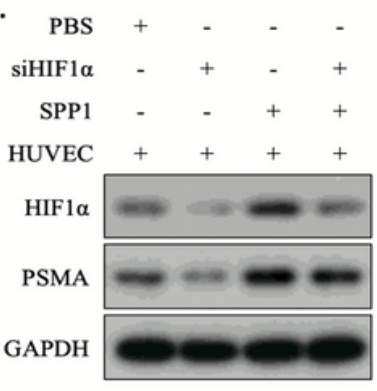

E.

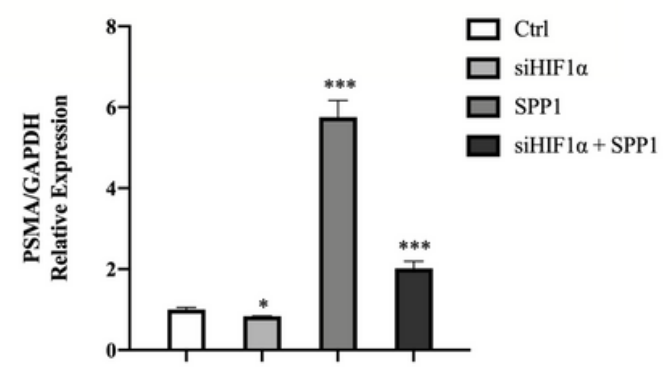

G.

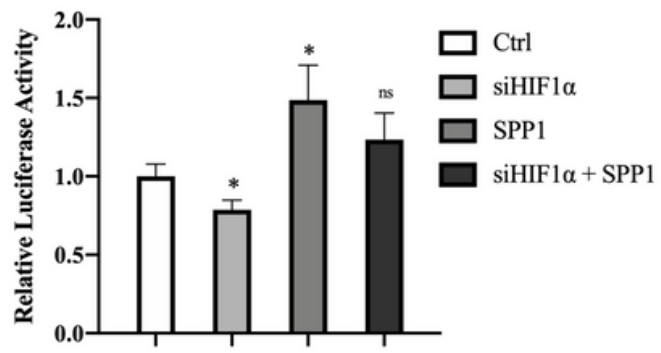

\section{Figure 3}

SPP1 promotes PSMA upregulation through transcription factor HIF1a. A-B Binding motif and DNA sequence of HIF1a on the promotor region of PSMA by JASPAR database; C The knockdown efficiency of siHIF1a in HUVEC by qRT-PCR; D-E The effect of SPP1 and HIF1a on the expression of PSMA by western blot and qRT-PCR; F ChIP assay of PSMA promotor with the HIF1a antibody in the presence of SPP1; $\mathbf{G}$ The effect of SPP1 and HIF1a on Dual-Luciferase reporter assay of PSMA promotor. 
A.

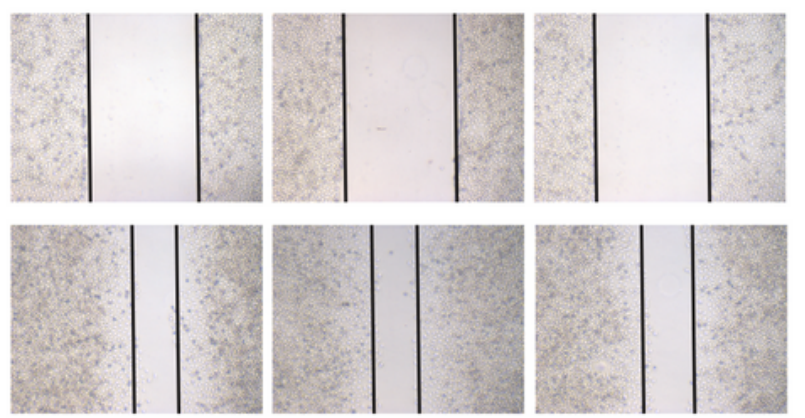

SPP1
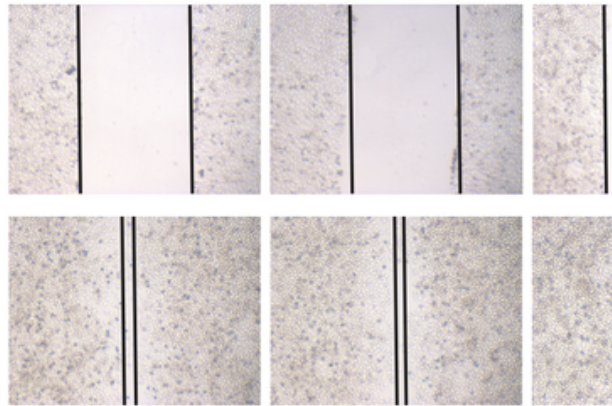

Oh

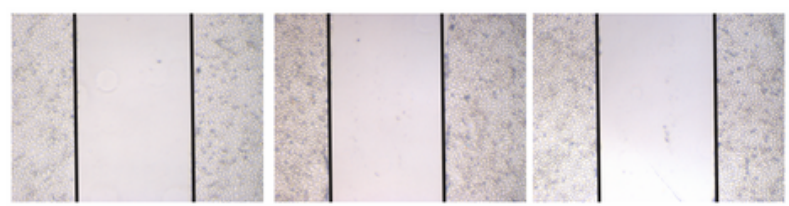

$48 \mathrm{~h}$

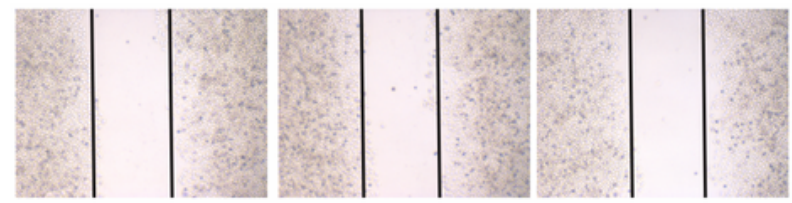

siHIF $1 \alpha+$ SPP1

Oh

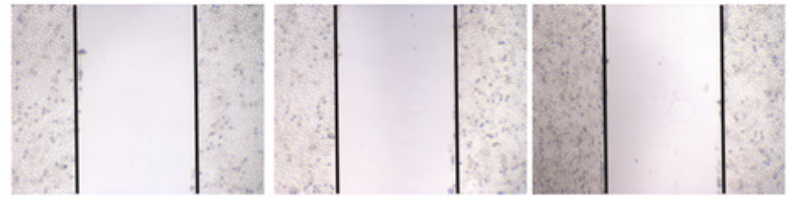

$48 \mathrm{~h}$

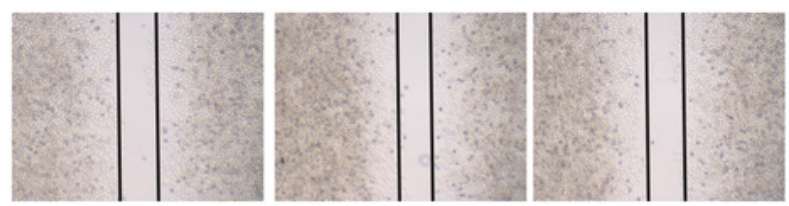

B.

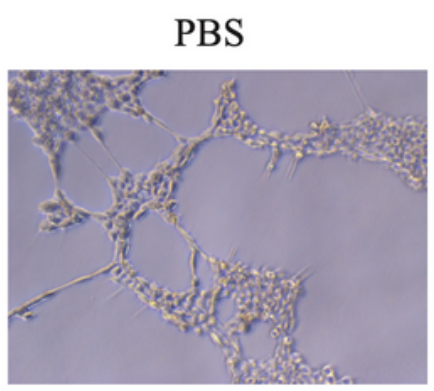

siHIF1 $\alpha$
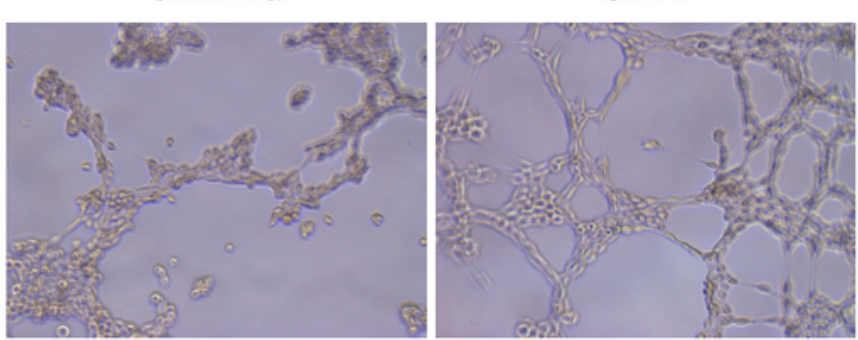

siHIF $1 \alpha+$ SPP1

C.

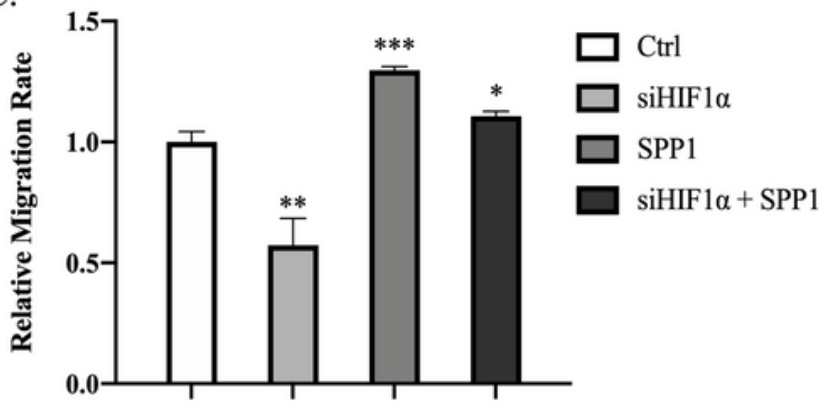

D.

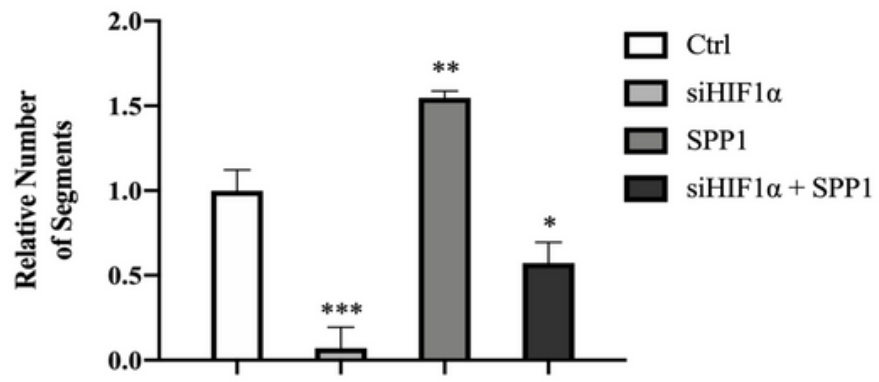

Figure 4

The ability of SPP1-regulated endothelial migration and tube formation could be blocked by knocking down HIF1a. A\&C The effect of SPP1 and HIF1a on the Scratch assay of HUVEC; B\&D The effect of SPP1 and HIF1a on tube formation assay of HUVEC. 
A.
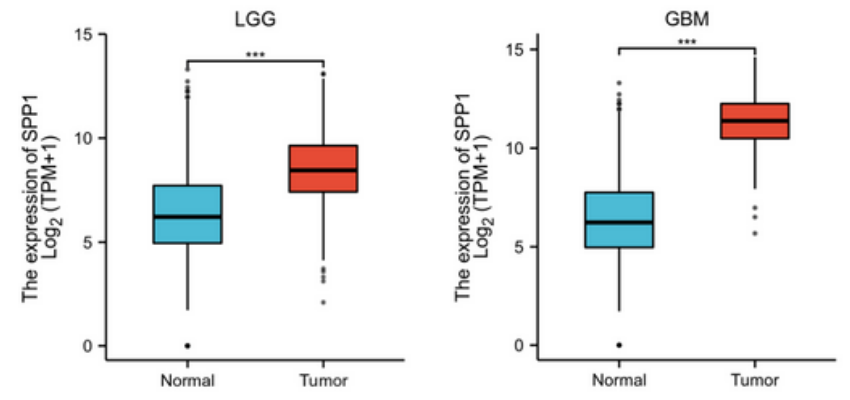

C.

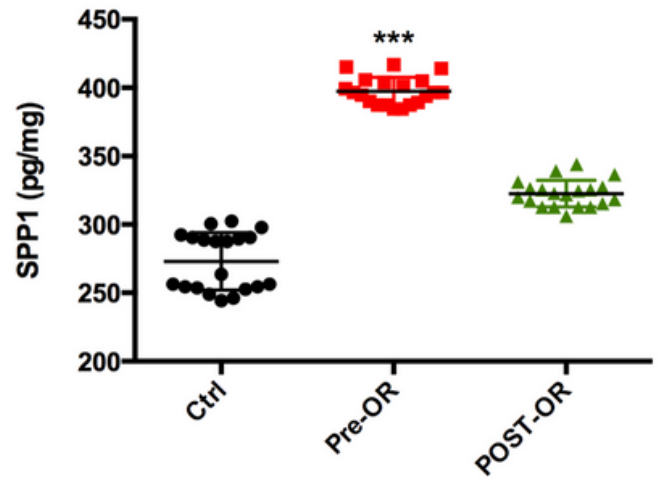

B.
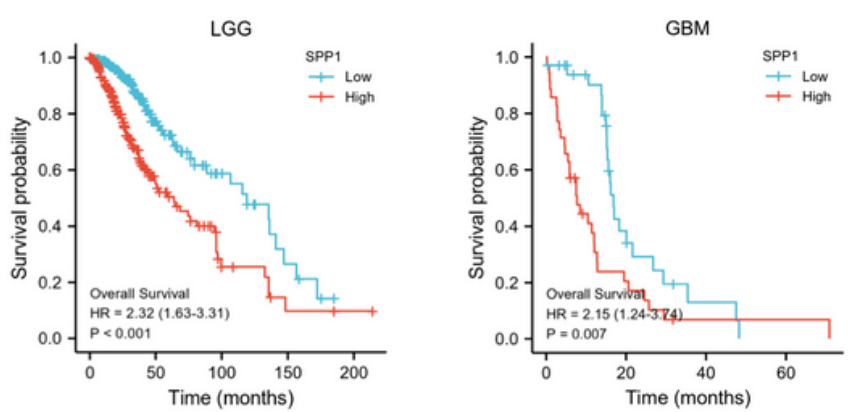

D.
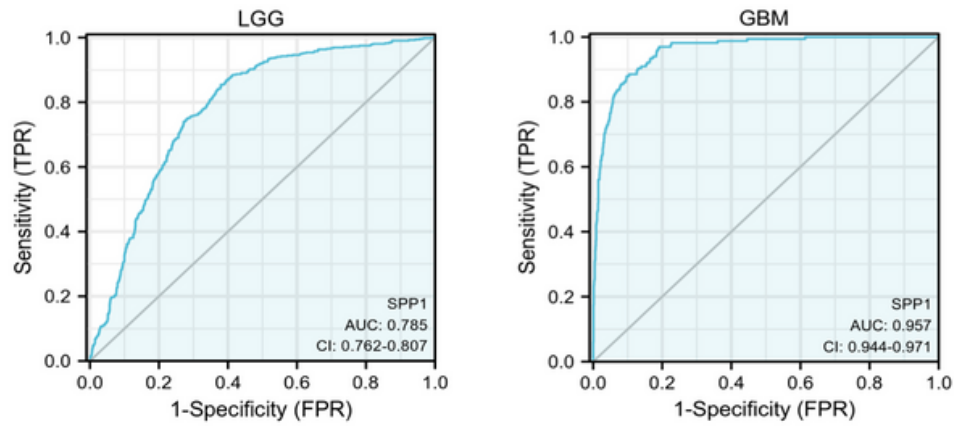

Figure 5

SPP1 is abundantly expressed in GBM and predicts poor prognosis. A The expression of SPP1 in LGG and GBM group by TCGA datasets; B The overall survival of SPP1 in LGG group and GBM group by TCGA datasets; C The concentration level of SPP1 in serum samples of normal human volunteers (Ctrl, $n=20)$, pre-operative GBM patients (Pre-OR, $n=20$ ) and post-operative GBM patients (Post-OR, $n=20$ ) by ELISA; D ROC curve analysis of SPP1 in LGG group and GBM group by TCGA datasets. 


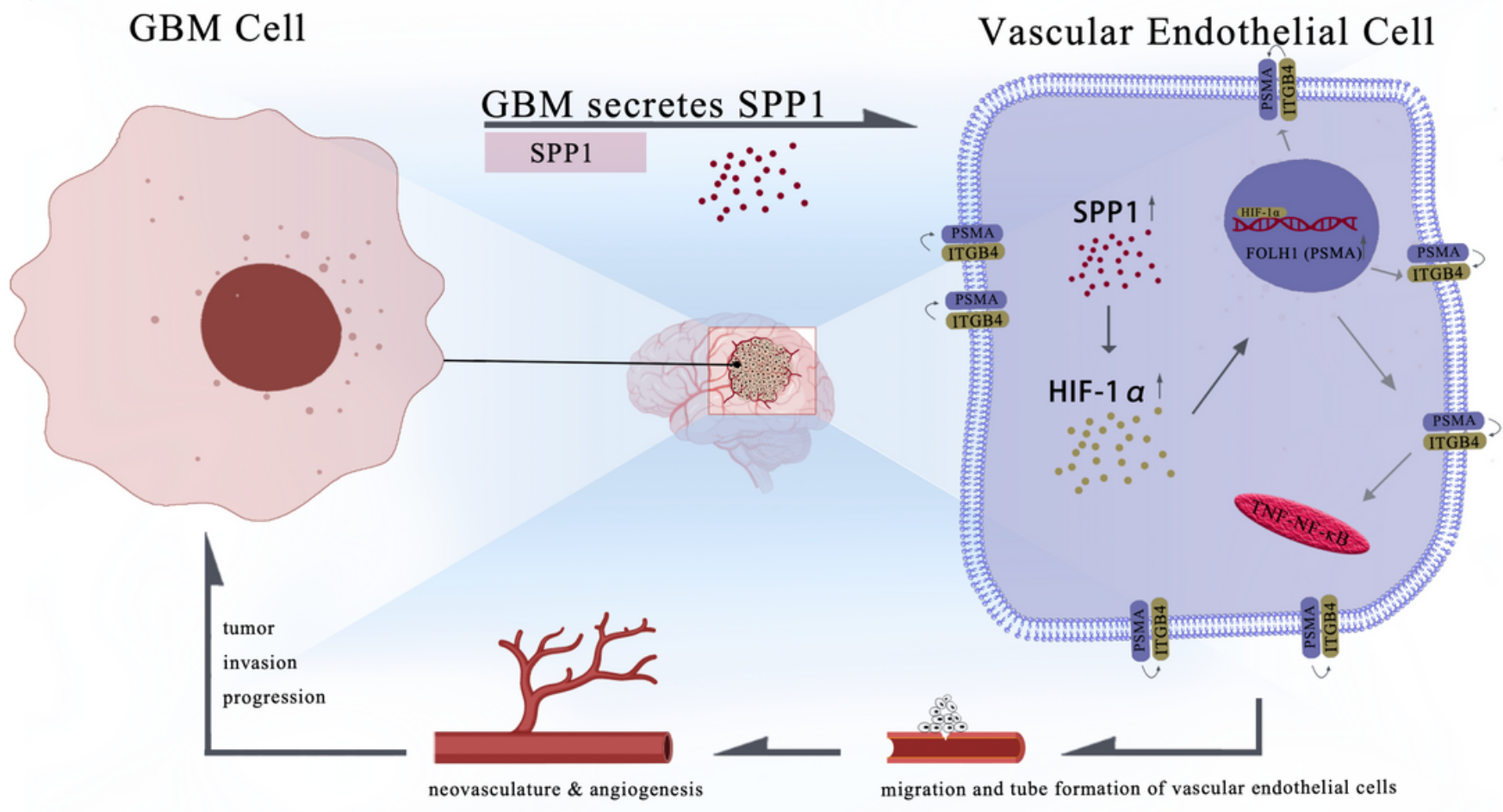

Figure 6

Schematic illustration summarizing the the function of SPP1-HIF1a-PSMA in GBM progression.

\section{Supplementary Files}

This is a list of supplementary files associated with this preprint. Click to download.

- Availabilityofdataandmaterials.pdf

- OriginalblotsofFig.2Dfiguretitleandlegendsadded.pdf

- OriginalblotsofFig.3Dfiguretitleandlegendsadded.pdf 